\title{
Consistent Approximations and Variational Description of Some Classes of Sliding Mode Control Processes
}

\author{
V. Azhmyakov ${ }^{1, a}$, Andrei Polyakov ${ }^{\mathrm{b}}$, Alex Poznyak ${ }^{\mathrm{a}}$ \\ ${ }^{a}$ Department of Control Automation, CINVESTAV, A.P. 14-740, CP 07000, Mexico \\ D.F., Mexico \\ ${ }^{b}$ NON-A, INRIA-LNE, Parc Scientifique de la Haute Borne 40, avenue Halley Bat.A, \\ Park Plaza, 59650Villeneuve d'Ascq, France
}

\begin{abstract}
This paper is devoted to constructive approximations and an alternative theoretic characterization of some classes of sliding mode control processes. We construct the consistent approximations of the di erential inclusions associated with the rst-order variable structures dynamics and also propose a variational description of the sliding mode control in the framework of an auxiliary Hamiltonian based formalism. A trajectory of the closed-loop systems can be then constructively speci ed as a result of a particular system optimization procedure applied to the original model. The presented approximations and variational description of the sliding mode-type control design can provide a new analytic basis for constructive numerical schemes and implementable control algorithms. The mathematical tool elaborated in our contribution constitutes a formal extension of the classic Fillipovs results.
\end{abstract}

Keywords: variable structure control systems, consistent approximations, Hamiltonian formalism, optimal control

\section{Introduction}

The study of various types of variable structures control processes in the framework of the general discontinuous dynamic models has gained lots of interest in recent years (see e.g., $[8,10,12,13,16,17,24,26,27,35,36$,

\footnotetext{
${ }^{1}$ Prof. V. Azhmyakov (corresponding author) Department of Control Automation, CINVESTAV, A.P. 14-740, CP 07000, Mexico City, Mexico tel./fax: +52 55-5747-3740; email: vazhmyakov@ctrl.cinvestav.mx
} 
$38,40,41,44,49])$. An important case of the above-mentioned systems is given by a class of the conventional first-order sliding mode control systems and the corresponding mathematical models expressed in the form of differential inclusions [43]. These sophisticated mathematical models are practically motivated by many modern engineering applications and constitute a valuable part of the recent progress in the systems theory. We refer to $[12,16,17,27,35,40,41,43,45,46,47,48,49,50]$ for the basic theoretic facts and also for some interesting real world applications of the sliding mode control techniques.

It is necessary to stress, that the control design approaches based on the traditional sliding mode technologies (see [43]) and on the high-order extensions $([26,35])$ are nowadays a mature and relative simple methodology for the constructive synthesis of several types of robust controllers and effective observers associated with the general nonlinearly affine dynamic systems. However, the formal mathematical techniques based on the approximation theory and on the modern variational analysis have not been sufficiently advanced to the variable structure systems, as well as to the corresponding computational algorithms. Recall that the general variational approach (the possibility to replace an original problem by an equivalent minimization problem) plays one of central roles in the applied functional and numerical analysis and constitute the valuable contributions of many prominent mathematicians (D. Bernoulli, L. Euler, J.L. Lagrange, K.F. Gauss, D. Hilbert and others). Our contribution pursues two main goals. Firstly, we are interested to provide a strong theoretic framework for consistent approximations of the specific differential inclusions induced by the sliding mode control systems. Secondly, we elaborate an adequate variational representation of the classic sliding mode dynamics and to deduce the associated state space formalism. And, it should be noted already at this point that a variational characterization of some classes of control systems can be essentially used in a concrete control synthesis procedure. Moreover, it can also leads to the new theoretic interpretations and numerically tractable approximations of the dynamic processes under consideration.

Our paper is organized as follows: Section 2 is devoted to some preliminary mathematical concepts and facts. We formalize our aims and introduce the set-valued representation that is associated with the specific (sliding mode type) closed-loop realization of the initial system. In Section 3 we propose two effective approximation techniques for the closed-loop variable structure systems under consideration. We introduce a so called $\beta$-system and also 
consider the first-order approximation approach. Section 4 deals with an equivalent variational description of the designed dynamic processes. We formulate a generic optimal control problem that constitutes a variational specification of the initial control system. In this section we formulate our main result and examine some possible special cases. Section 4 also discusses shortly an application of the developed variational technique to the attainability analysis. In Section 5 we extend the obtained theoretic results by the corresponding numerical issues and consider a computational example. Section 5 also discusses shortly an application of the developed variational technique to the attainability and finite time stability analysis. Section 6 summarizes the paper.

\section{Problem Formulation and Some Preliminaries}

\subsection{Systems Description}

Consider the nonlinearly affine control system

$$
\begin{aligned}
& \dot{x}(t)=a(x(t))+b(x(t)) u(t) \text { a.e. on }\left[0, t_{f}\right], \\
& x(0)=x_{0},
\end{aligned}
$$

where $x_{0} \in \mathbb{R}^{n}$ is a given initial state. The functions $a: \mathcal{R} \rightarrow \mathbb{R}^{n}$ and $b: \mathcal{R} \rightarrow \mathbb{R}^{n \times m}$ are assumed to be sufficiently many times differentiable on an open set $\mathcal{R} \subseteq \mathbb{R}^{n}$. From the general existence/uniqness theory for nonlinear ordinary differential equations (see e.g., $[29,34,41]$ ) it follows that for every admissible control $u(\cdot) \in \mathbb{L}_{m}^{\infty}\left(0, t_{f}\right)$, where $\mathbb{L}_{m}^{\infty}\left(0, t_{f}\right)$ is the Lebesgue space of all measurable essentially bounded functions, the initial value problem (1) has a unique absolutely continuous solution $x(\cdot)$. Let us further consider the case $m<n$. We also assume that the rank of $\operatorname{span}\left\{F_{1}(x), \ldots, F_{m}(x)\right\}$, where $F_{j}(x)$ are vector-columns of the matrix $b(x)$, is equal to $m \in \mathbb{N}$.

The specific class of the affine dynamical models introduced above represents a wide and important family of general dynamic systems. Various control systems of the type (1) arise in the control of mechanical systems, electrical circuits and aircrafts [16, 32, 34, 43, 47]. The switched and hybrid affine systems, where different models are coupled together, are considered in [2] (see also $[6,7,8]$ and the literature herein). Systems of the type (1) constitutes the main mathematical object of the variable structure control 
methodology (see e.g., [12, 32, 43]). The sliding mode character of the dynamic system (1) is determined by the following specific feedback control functions:

$$
w(x):=\tilde{w}(\sigma(x)),
$$

where $\tilde{w}: \mathbb{R}^{m} \rightarrow \mathbb{R}^{m}$ is a bounded measurable (feedback) control function and $\sigma: \mathbb{R}^{n} \rightarrow \mathbb{R}^{m}$ is a continuously differentiable $m$-valued function. The classic sliding mode dynamic process associated with (1) usually incorporates a "sliding manifold", $\mathcal{S}:=\left\{x \in \mathbb{R}^{n} \mid \sigma(x)=0\right\}$ that determines the main feedback-type control strategy in the following sense: a trajectory $x(\cdot)$ generated by the closed-loop system (1)-(2) possesses the following property

$$
\sigma(x(t))=0, \forall t \geq T_{s l} \in \mathbb{R}_{+}, \quad T_{s l}<t_{f} .
$$

Let us note that the composite function $u_{w}(t) \equiv w(x(t))$, where $x(\cdot)$ is a trajectory of the resulting system (1)-(2), belongs to the space $\mathbb{L}_{m}^{\infty}\left(0, t_{f}\right)$. This is an easy consequence of the boundedness of $\tilde{w}(\cdot)$ and absolute continuity of the solution $x(\cdot)$ to the closed-loop realization of (1)-(2). Note that the main assumptions introduced above make it possible to incorporate some classes of discontinuous closed-loop systems into the considered framework. In that case the solution concept for (1)-(2) needs an additional justification. An adequate constructive definition of a solution here can be considered, for example, in the sense of the well-knowm Filippov approach [25, 38, 43]. Let us also recall that the more general (higher order) sliding mode control approach to systems with a relative degree $l \in \mathbb{N}$ is related to a family of the smooth functions of the type $\bar{\sigma}(t, x)$ and to a specific form of the feedback control law. In this case the traditional sliding mode formalism is defined by $(l-1)$ derivatives of the function $\bar{\sigma}(\cdot, \cdot)$ (see e.g., $[12,26,35])$.

Evidently, the sliding mode control systems are characterized by a specific mechanism of the states transitions. This prescribed switching mechanism is determined by a discontinuous feedback control (2) that is implemented for all $0<t<T_{s l}$. The generic sliding condition $\sigma(x(t))=0$ for $t \geq T_{s l}$ evidently implies the inclusion

$$
\dot{x}(t) \in \mathcal{T}_{\mathcal{S}}(x(t))
$$

for all $t \geq T_{s l}$. By $\mathcal{T}_{\mathcal{S}}(z)$ we denote here the tangent space of $\mathcal{S}$ at $z \in \mathcal{S}$. In this paper we restrict our consideration to the linear-affine sliding surfaces $\mathcal{S}$ defined by $\sigma(x)=S x+c$, where $S \in \mathbb{R}^{m \times n}, c \in \mathbb{R}^{m}$. The last inclusion can 
be easily rewritten in the following form

$$
\nabla \sigma(x(t))\left[a(x(t))+b(x(t)) w_{e q}(x(t))\right]=0, \forall t \geq T_{s l}
$$

that determines explicitly the celebrated equivalent control $w_{e q}(\cdot)$ in the case of an invertible matrix $\nabla \sigma(x) b(x)$

$$
w_{e q}(x):=-(\nabla \sigma(x) b(x))^{-1}[\nabla \sigma(x) a(x)]=-(S b(x))^{-1}[S a(x)] .
$$

We use here the standard notation $\nabla \sigma(x)$ for a gradient of $\sigma(x)$ at $x \in \mathcal{S}$ and additionally assume the invertibility of the matrix $S b(x)$ for all $x \in \mathbb{R}^{n} \backslash 0$. Let us denote $u_{e q}(t):=w_{e q}(x(t))$. The complete sliding mode control law can now be written as follows:

$$
u_{s l}(t)=\left\{\begin{array}{l}
u_{w}(t), \text { if } t<T_{s l} \\
u_{e q}(t), \text { if } t \geq T_{s l}
\end{array}\right\}
$$

Clearly, this form of the designed input can be formally obtained only under the assumption of a known trajectory of the system. We call system (1) with the implemented control $u_{s l}(\cdot)$ the closed-loop realization of $(1)$.

\subsection{The Set Valued Based Approach}

In this paper, we propose a new variational representation of the sliding behavior associated with this model that incorporates the classic Hamiltonian formalism. Resulting from this Hamiltonian-like representation in an extended state space the realized sliding trajectory of (1) can be constructively described as a solution to a specific auxiliary optimal control problem. Moreover, the representation mentioned above makes it possible to calculate the equivalent control $u_{e q}(\cdot)$ as a time depending function in (4).

As mentioned above, the closed-loop realization (1)-(4) is in general determined by ordinary differential equations with discontinuous right hand sides. The discontinuity effect is caused by the given class of the of the feedback control functions $w(\cdot)$ and $w_{e q}(\cdot)$. In a concrete situation a suitable sliding mode-based control design needs to posses some strong stability properties. This stability requirement is usually related the classic Lyapunov or finitetime stability concepts (see $[12,15,31,37]$ ). We consider the corresponding closed-loop system (1) as a special case of a general differential equation with

discontinuous right hand side. This modeling framework makes it possible to 
apply the celebrated Filippov approach $[8,25,16,21,39,38])$ and to study the associated differential inclusion of the following form

$$
\dot{\tilde{x}}(t) \in \mathcal{K}[a, b](x(t)) \text { a.e. on }\left[0, t_{f}\right],
$$

where $x(0)=x_{0}$ and

$$
\mathcal{K}[a, b](x):=\overline{\operatorname{co}}\left\{\lim _{j \rightarrow \infty}\left[a\left(x_{i}\right)+b\left(x_{i}\right) w\left(x_{i}\right)\right] \mid x_{i} \rightarrow x, x_{i} \notin \mathcal{P}\right\}
$$

Here $\mathcal{P} \subset \mathbb{R}^{n}$ is a set of measure zero. Our aim now is with the development of an adequate constructive characterization of the sophisticated closed-loop realization of (1). Such an equivalent description of the initial mathematical model (5) can provide a new theoretic basis for some effective control design procedures. The expected equivalent or approximative description of (5) can naturally be separated into two parts, namely, for $t<T$ (the Part I) and for $t \geq T$ (the Part II). For the mathematical description of Part I one can use, for example, the set-valued approximation techniques proposed in [10]. Let us shortly discuss this newly elaborated approximative tool. The structure of the feedback control (2) motivates the consideration of the boxtype admissible controls set $\mathcal{U}$

$$
\begin{aligned}
\mathcal{U} & :=\left\{v(\cdot) \in \mathbb{L}_{m}^{\infty}\left(0, t_{f}\right) \mid v(t) \in U \text { a.e. on }\left[0, t_{f}\right]\right\}, \\
U & :=\left\{u \in \mathbb{R}^{m}: v_{-}^{j} \leq u_{j} \leq v_{+}^{j}, j=1, \ldots, m\right\},
\end{aligned}
$$

where $v_{-}^{j}, v_{+}^{j}, j=1, \ldots, m$ are some constants. Using the general form (5), the affine system (1)-(4) can now be rewritten in the equivalent form as the following differential inclusion

$$
\begin{aligned}
& \dot{x}(t) \in a(x(t))+b(x(t)) U, \\
& x(0)=x_{0},
\end{aligned}
$$

where $a(t, x(t))+b(t, x(t)) U$ is the orientor field associated with (1)-(4) for a given compact and convex set $U$. We denote (6) as an inclusion generated by the closed-loop realization (1)-(4). The original differential inclusion (8) with affine structure admits a conventional parametrization in the form of a relaxed control system (the Gamkrelidze system [28])

$$
\begin{aligned}
& \dot{\eta}(t)=a(\eta(t))+\sum_{j=1}^{n+1} \alpha^{j}(t) b(\eta(t)) u^{j}(t) \text { a.e. on }\left[0, t_{f}\right], \\
& \eta(0)=x_{0}, \alpha(\cdot) \in \Lambda(n+1),
\end{aligned}
$$


where $\alpha(\cdot):=\left(\alpha^{1}(\cdot), \ldots, \alpha^{n+1}(\cdot)\right)^{T}, \quad u^{j}(\cdot) \in \mathcal{U} \quad \forall j=1, \ldots, n+1$ and $\Lambda(n+1):=\left\{\alpha(\cdot) \mid \alpha^{j}(\cdot) \in \mathbb{L}_{1}^{1}\left(0, t_{f}\right), \alpha^{j}(t) \geq 0, \sum_{j=1}^{n+1} \alpha^{j}(t)=1, t \in\left[0, t_{f}\right]\right\}$,

is a set of admissible multipliers $\alpha(\cdot)$. Let $\eta(\cdot)$ be a solution to the Gamkrelidze system (7) generated by an admissible controls $u(\cdot) \in \mathcal{U}$ and multipliers $\alpha(\cdot) \in \Lambda(n+1)$. We refer to $[1,8,10,14,21,22]$ for the exact results related to the explicit Gamkrelidze-factorization of the convex-valued differential inclusion (6). This result can be shortly summarized as follows

Proposition 1. A function $x(\cdot)$ is a solution of the inclusion (6) if and only if it is a solution of the corresponding Gamkrelidze system (7).

Note that Proposition 1 is a direct consequence of the so called Filippov Selection Lemma (see e.g., [1, 21, 30, 39]).

\section{Constructive Approximations of the Sliding Mode Dynamics}

\subsection{The Approximating $\beta$-System}

Our aim now is to construct an approximative approach to the initial dynamic model (6). Let us assume that for a given number $\epsilon_{M}>0$ (the prescribed accuracy) and for every element $\omega \in U$ there exist a point $\omega^{k} \in U_{M}$ (the discrete approximations of the given control set $U$ ) such that

$$
|| \omega-\omega^{k} \|_{\mathbb{R}^{m}}<\epsilon_{M}
$$

Following the theoretic construction developed in [10], we introduce the so called $\beta$-system

$$
\begin{aligned}
& \dot{z}(t)=a(z(t))+\sum_{k=1}^{M} \beta^{k}(t)\left(b(z(t)) \omega^{k}\right) \text { a.e. on }\left[0, t_{f}\right], \\
& z(0)=x_{0},
\end{aligned}
$$

where $\beta^{k}(\cdot)$ are elements of $\mathbb{L}_{m}^{\infty}\left(0, t_{f}\right)$ such that

$$
\beta^{k}(t) \geq 0, \quad \sum_{k=1}^{M} \beta^{k}(t)=1 \quad \forall t \in\left[0, t_{f}\right] .
$$


The introduced vector $\beta_{M}(\cdot):=\left(\beta^{1}(\cdot), \ldots, \beta^{M}(\cdot)\right)^{T}$ is called a $\beta$-control. The set of admissible $\beta$-controls is denoted by $\aleph(M)$. Let $\mathbb{W}_{n}^{1,1}\left(0, t_{f}\right)$ be the Sobolev space and $\mathbb{C}_{n}\left(0, t_{f}\right)$ be the space of continuous functions. Consider a sequence of approximations $U_{M}$ corresponding to the given accuracy $\epsilon_{M}$ such that $\lim _{M \rightarrow \infty} \epsilon_{M}=0$. The main result from [10] shows that the $\beta$-systems (8) generated by $U_{M}$ are consistent approximations of the Gamkrelidze system associated with (6).

Theorem 1. Consider the initial system (6) and a solution $\eta(\cdot)$ of the corresponding Gamkrelidze system (7). Then there exists a sequence $\left\{\beta_{M}(\cdot)\right\}$ from $\aleph(M)$ of the $\beta$-controls and the corresponding sequence $\left\{z_{M}^{\beta}(\cdot)\right\}$ of solutions of $\beta$-systems such that $z_{M}^{\beta}(\cdot)$ approximate the solution $y(\cdot)$ in the following senses $\lim _{M \rightarrow \infty}\left\|z_{M}^{\beta}(\cdot)-y(\cdot)\right\|_{\mathbb{C}_{n}\left(0, t_{f}\right)}=0$, and

$$
\lim _{M \rightarrow \infty}\left\|z_{M}^{\beta}(\cdot)-y(\cdot)\right\|_{\mathbb{W}_{n}^{1,1}\left(0, t_{f}\right)}=0 .
$$

Moreover, every $z_{M}^{\beta}(\cdot)$ is also a solution of the differential inclusion

$$
\dot{z}_{M}^{\beta}(t) \in a\left(z_{M}^{\beta}(t)\right)+b\left(z_{M}^{\beta}(t)\right) \times U \text { a.e. on }\left[0, t_{f}\right],
$$

where $z(0)=x_{0}$.

Theorem 1 provides an effective approximative approach to differential inclusion (8) and to the initial closed-loop realization (1)-(4) on the time interval $[0, T)$. Recall that this behavior of the complete sliding mode dynamics was denoted as a Part I. For the practically oriented approximative methods and for the concrete control synthesis procedures one now can eliminate the exact Gamkrelidze system (7) from consideration and use the auxiliary $\beta$-systems (8) or the approximative differential inclusion (9). Note that the method based on the $\beta$-system (8) was effectively applied in [10] to problems of optimal control design in some particular cases of variable structure dynamics.

\subsection{First-Order Approximations}

Let us now discuss the Part II of the complete sliding mode dynamic behavior and concentrate on the corresponding approximative techniques for this part of the control process. Consider the time interval $\left[T_{s l}, t_{f}\right]$ and introduce a bounded measurable variation $\delta u(\cdot)$ of the given control $u_{e q}(t)$. 
The variation of the state variable generated by $u_{e q}(\cdot)+\delta u(\cdot)$ is equal to $x(\cdot)+\delta x(\cdot)$. Evidently, the original (non-disturbed) control $u_{e q}(\cdot)$ and the realized trajectory $x(\cdot)$ determine the reference sliding dynamics of the closedloop system (1). Using the standard first-order argument and linearization techniques (see e.g., [29, 34, 41], we can easily derive the variational differential equation associated with the system (1) closed by the equivalent control

$$
\begin{aligned}
& \dot{\delta x}(t)=\left[\frac{\partial a}{\partial x}(x(t))+\sum_{j=1}^{m} \frac{\partial F_{j}}{\partial x}(x(t)) w_{e q}^{j}(x(t))\right] \delta x(t)+b(x(t)) \delta u(t), \\
& \delta x(0)=0
\end{aligned}
$$

Note that (10) is considered on the interval $\left[0, t_{f}\right]$ and $w_{e q}^{j}(x), j=1, \ldots, m$ denotes the component of $w_{e q}(x)$.

Let $\mathcal{U}_{\mathcal{S}}$ be a subset of $\mathcal{U}$ such that $x(t)+\delta x(t) \in \mathcal{S}$ for all $t \geq T_{\delta}$ with $T_{\delta}<t_{f}$. From the general results of the variable structure systems theory it follows that the set $\mathcal{U}_{\mathcal{S}}$ is nonempty (see e. g., $[12,35,38]$ and the references therein). The following result is an immediately consequence of the classic equivalent control concept [43].

Theorem 2. Let all conditions from Section 2 and Section 3 are satisfied and $u_{e q}(\cdot)+\delta u(\cdot) \in \mathcal{U}_{\mathcal{S}}$ for a bounded measurable $\delta u(\cdot)$. Then we have $\delta x(t) \in$ $\mathcal{S}$ for all $t \geq T_{\delta}$.

Proof: From the definition of the set $\mathcal{U}_{\mathcal{S}}$ and (3) we easily deduce the following

$$
S\left[a(x(t)+\delta x(t))+b(x(t)+\delta x(t))\left(w_{e q}(x(t))+\delta u(t)\right)\right]=0
$$

for all $t \geq T_{\delta}$. Since $u_{e q}(\cdot)=w_{e q}(x(t))$ is an equivalent control for the original system (1), we have

$$
S\left[a(x(t))+b\left(x(t) w_{e q}(x(t))\right)=0 \quad \forall t \geq T_{\delta} .\right.
$$

Using the conventional first-order argument, we easily obtain

$$
\left.S\left[\left(\frac{\partial a}{\partial x}(x(t))+\sum_{j=1}^{m} \frac{\partial F_{j}}{\partial x}(x(t)) w_{e q}^{j}(x(t))\right) \delta x(t)+b(x(t)) \delta u(t)\right)\right]=0
$$


for $t \geq T_{\delta}$. The last relation shows that the states $\delta x(t)$ associated with the linearized system (10) with $\delta u(\cdot)$ belong to $\mathcal{S}$ for all $t \geq T_{\delta}$. The proof is finished.

From Theorem 2 it follows that the linear-affine sliding surface $\sigma(\cdot)$ also constitutes a sliding surface for the linearized trajectory $\delta x(\cdot)$. Let $x^{u_{e q}+\delta u}(\cdot)$ be the solution of (1) generated by and admissible $u_{e q}(\cdot)+\delta u(\cdot)$. By $x^{u_{e q}}(\cdot)$ we denote a solution to (1) associated with $u_{e q}(\cdot)$. Assume that $\delta x(\cdot)$ solves (10) closed by an admissible $\delta u(\cdot)$ such that $u(\cdot)+\delta u(\cdot) \in \mathcal{U}_{\mathcal{S}}$. The consistency of linear approximations given by (10) can be established using the general continuity result from [11].

Theorem 3. Assume that the initial system (1) satisfies all technical assumptions from Section 2 and Section 3. Then there exists a function $o: \mathbb{R}_{+} \rightarrow \mathbb{R}_{+}$such that $s^{-1} o(s) \rightarrow 0$ as $s \downarrow 0$ and

$$
\left\|x^{u_{e q}+\delta u}(\cdot)-\left(x^{u_{e q}}(\cdot)+\delta x(\cdot)\right)\right\|_{\mathbb{L}_{n}^{\infty}} \leq o\left(\|\delta u(\cdot)\|_{\mathbb{L}_{m}^{\infty}}\right)
$$

for all $u_{e q}(\cdot)+\delta u(\cdot) \in \mathcal{U}_{\mathcal{S}}$.

Proof: From the celebrated Gronwall Lemma and the corresponding comparison result (see $[14,41]$ ) with the comparison functions

$$
\xi(t):=x^{u_{e q}}(t)+\delta x(\cdot)(t), \quad \psi(t, x):=a(x)+b(t) u+\delta u(t),
$$

we obtain

$$
\begin{aligned}
& \left\|x^{u_{e q}+\delta u}(\cdot)-\left(x^{u_{e q}}(\cdot)+\delta x(\cdot)\right)\right\|_{\mathbb{L}_{n}^{\infty}} \leq e^{C} \int_{T_{s l}}^{t_{f}} \| \dot{x}^{u_{e q}}(t)+\dot{\delta x}(t)- \\
& a\left(x^{u_{e q}}(t)+\delta x(t)\right)-b(t)\left(u_{e q}(t)+\delta u(t)\right) \| d t= \\
& e^{C} \int_{T_{s l}}^{t_{f}} \|\left\langle\left(a_{x}\left(x^{u_{e q}}(t)\right), b(t)\right),(\delta x(t), \delta u(t))\right\rangle- \\
& {\left[a\left(x^{u_{e q}}(t)+\delta x(t)\right)+b(t) \delta u(t)-a\left(x^{u_{e q}}(t)\right] \| d t\right.}
\end{aligned}
$$

for a constant $C$. Using the component-wise variant of the classic Mean Value Theorem, we conclude that

$$
\begin{aligned}
& \left.a_{i}\left(x^{u_{e q}}(t)\right)+\delta x(t)\right)+b(t)\left(u_{e q}(t)+\delta u(t)\right)-\left(a_{i}\left(x^{u_{e q}}(t)\right)+b(t) u_{e q}(t)\right)= \\
& \left.\left\langle\left(a_{i}\right)_{x}\left(x^{u_{e q}}(t)+\nu_{i}(t)\right), b(t)\right)(\delta x(t), \delta u(t))\right\rangle
\end{aligned}
$$


for a suitable bounded function $\nu(\cdot)$ and with $i=1, \ldots, n, j=1, \ldots, m$. The differentiability of $a(\cdot)$ implies the Lipschitz continuity of this function on a bounded set $\mathcal{R}$. The last observation causes the existence of a function $o_{1}: \mathbb{R}_{+} \rightarrow \mathbb{R}_{+}$such that $s^{-1} o_{1}(s) \rightarrow 0$ as $s \downarrow 0$ and

$$
\begin{aligned}
& \|\left\langle\left(a_{x}\left(x^{u_{e q}}(t)\right), b(t)\right),(\delta x(t), \delta u(t))-\right. \\
& {\left[a\left(x^{u_{e q}}(t)+\delta x(t)\right)+b(t) \delta u(t)-a\left(x^{u_{e q}}(t)\right)\right] \leq o_{1}\left(\|\delta u(\cdot)\|_{\mathbb{L}_{m}^{\infty}}\right)}
\end{aligned}
$$

for all $t$ from the given interval $\left[T_{\text {sl }}, t_{f}\right]$. From (11) we finally deduce the expected estimation

$$
\left\|x^{u_{e q}+\delta u}(\cdot)-\left(x^{u_{e q}}(\cdot)+\delta x(\cdot)\right)\right\|_{\mathbb{L}_{n}^{\infty}} \leq o\left(\|\delta u(\cdot)\|_{\mathbb{L}_{m}^{\infty}}\right)
$$

with $o(s):=e^{C} o_{1}(s)$. The proof is finished.

We refer to [11] for the proof of a general version of Theorem 3.

\section{An Optimal Control Based Characterization of the Sliding Mode Behavior}

\subsection{The Hamiltonian Formalism for Sliding Mode Processes}

The sliding property of solutions $\delta x(\cdot)$ to (10) make it possible to derive a Hamiltonian formalism associated with the closed-loop realization of the original nonlinearly affine system (1). This equivalent representation makes a sense for the Part II of the complete systems trajectory, namely, for the time interval $\left[T_{s l}, t_{f}\right]$. Consider a restriction of the linear system (10) on $\left[T, t_{f}\right]$, where $T:=\max \left\{T_{s l}, T_{\delta}\right\}$. We also put $\delta x(T)=0$. Let $\Phi(\cdot)$ be a the fundamental matrix on $\left[T, t_{f}\right]$ solution a.e. of

$$
\begin{aligned}
& \dot{\Phi}(t)=\left[\frac{\partial a}{\partial x}(x(t))+\sum_{j=1}^{m} \frac{\partial F_{j}}{\partial x}(x(t)) w_{e q}^{j}(x(t))\right] \Phi(t), \\
& \Phi(T)=I .
\end{aligned}
$$

Here $I$ is the unit matrix. We are now ready to formulate the main theoretic result of our contribution. 
Theorem 4. Consider the closed-loop realization of (1)-(4) on the "sliding" time interval $\left[T, t_{f}\right]$. Then there exists an absolutely continuous function (the adjoint vector) $p(\cdot), p(t) \in \mathbb{R}^{n}$ such that $\left(u_{e q}(\cdot), x(\cdot), p(\cdot)\right)$ are solutions a.e. on $\left[T, t_{f}\right]$ of the following Hamiltonian system

$$
\begin{aligned}
& \dot{x}(t)=\frac{\partial H}{\partial p}\left(u_{e q}(t), x(t), p(t)\right), \\
& \dot{p}(t)=-\frac{\partial H}{\partial x}\left(u_{e q}(t), x(t), p(t)\right), \\
& \left.\frac{\partial H}{\partial u}\left(u_{e q}(t), x(t), p(t)\right)\right)=0,
\end{aligned}
$$

where $H(u, x, p):=\langle p, a(x)+b(x) u\rangle$ is the pseudo-Hamiltonian and $\langle\cdot, \cdot\rangle$ being the inner product.

Proof: Note that the first equation from (12) is a simple consequence of the linear structure of the Hamiltonian. Consider (11) for such bounded measurable functions $\delta u(\cdot)$ that guarantee $u_{e q}(\cdot)+\delta u(\cdot) \in \mathcal{U}_{\mathcal{S}}$. From Theorem 2 it follows that the dimension of the linear space

$$
\left\{\delta x(\cdot) \in \mathbb{L}_{m}^{\infty}\left(0, t_{f}\right) \mid \Phi\left(t_{f}\right) \int_{T}^{t_{f}} \Phi^{-1}(t) b(x(t)) \delta u(t) d t\right\}
$$

that contains the set

$$
\left\{\delta x(\cdot) \mid \Phi\left(t_{f}\right) \int_{T}^{t_{f}} \Phi^{-1}(t) b(x(t)) \delta u(t) d t\right\}
$$

is equal $m$. Therefore, there exists a vector $\rho \in \mathbb{R}^{n}$ such that

$$
\rho^{T} \Phi\left(t_{f}\right) \Phi^{-1}(t) b(x(t))=0
$$

for almost all $t \in\left[T, t_{f}\right]$. We now introduce the function $p(\cdot)$ by setting $p(t):=\rho^{T} \Phi\left(t_{f}\right) \Phi^{-1}(t)$. The definition of $p(\cdot)$ implies that this function is a solution to the adjoint system

$$
\dot{p}(t)=-p(t)\left[\frac{\partial a}{\partial x}(x(t))+\sum_{j=1}^{m} \frac{\partial F_{j}}{\partial x}(x(t)) w_{e q}^{j}(x(t))\right]
$$

Moreover, it satisfies almost everywhere the equality $p(t) b(x(t))=0$ and we get the second and the third equations in (12). The proof is completed. 
Theorem 4 gives a variational characterization of the sliding dynamics given by $\left(u_{e q}(\cdot), x(\cdot)\right)$. Evidently, this theorem is similar to so called weak Pontryagin Maximum Principle in optimal control (see [14, 18, 22, 28]). The restriction of the sliding trajectory $x(\cdot)$ on the interval $\left[T, t_{f}\right]$ satisfies so called weak Maximum Principle (see e.g., [22]) with the Hamiltonian $H(u, x(t), p(t))$. Moreover, similar to the optimal control theory (see e.g., $[14,18,22,41])$ one can deduce some simple consequences of (12). For example, we have

$$
\begin{aligned}
& \frac{d H}{d t}\left(u_{e q}(t), x(t), p(t)\right)=0+\frac{\partial H}{\partial x}\left(u_{e q}(t), x(t), p(t)\right)[a(x(t))+ \\
& \left.b(x(t)) u_{e q}(t)\right]+\frac{\partial H}{\partial p}\left(u_{e q}(t), x(t), p(t)\right) \dot{p}(t)=-\dot{p}(t)[a(x(t))+ \\
& \left.b(x(t)) u_{e q}(t)\right]+\left[a(x(t))+b(x(t)) u_{e q}(t)\right] \dot{p}(t)=0
\end{aligned}
$$

for a solution $\left(u_{e q}(\cdot), x(\cdot), p(\cdot)\right)$ of $(12)$ on $\left[T, t_{f}\right]$. Evidently, (13) also specifies the given manifold $\mathcal{S}$. We have

$$
\begin{aligned}
& \tilde{H}(t):=\left\langle p(t), a(x(t))+b\left(x(t) w_{e q}(x(t))\right\rangle=\right. \\
& \left\langle p(t), a(x(t))-b\left(x(t)(S b(x))^{-1}[S a(x)]\right\rangle=\mathrm{const}\right.
\end{aligned}
$$

One can interpret the derived relations (13) and (14) as a generalized "energetic" description of the sliding dynamics $\left(u_{e q}(\cdot), x(\cdot)\right)$ and the manifold $\mathcal{S}$. This interpretation can be formalized as follows

$$
\begin{aligned}
& \dot{x}(t)=\frac{\partial H}{\partial p}\left(u_{e q}(t), x(t), p(t)\right), \\
& \dot{p}(t)=-\frac{\partial H}{\partial x}\left(u_{e q}(t), x(t), p(t)\right), \\
& \tilde{H}(t)=H\left(u_{e q}(t), x(t), p(t)\right)=\text { const, } t \in\left[T, t_{f}\right] .
\end{aligned}
$$

It is necessary to stress that the sliding manifold $\sigma(\cdot)$ is in general an algebraic invariant of the initial closed-loop system (1)-(4). Hence $\nabla \sigma(x(t)) \dot{x}(t)=0$ holds only for $x \in \mathcal{S}$. In contrast to that case, the Hamiltonian "sliding manifold" determined by $H(u, x, p)$ - const $=0$ is a first integral of the extended system (12) and the relation

$$
\left\langle\nabla_{(x, p)} H\left(u_{e q}(t), x(t), p(t)\right)\left(\dot{x}^{T}(t), \dot{p}^{T}(t)\right)^{T}\right\rangle=0
$$

is true in an open subset of $\mathbb{R}^{2 n}$. Let us also note that the asymptotic stability and the invariant property of the "Hamiltonian" manifold determined by 
$\tilde{H}^{-1}$ (const) can be easier stated as the same property of $\mathcal{S}$ in (1)-(4). The corresponding stability/invariance conditions for (15) are given by the known Ascher-Chin-Reich Theorem [4].

We now consider a simple example associated with the obtained main result.

Example 1. Let $n=2, m=1$ and $\mathcal{S}=(0,1)$. Assume that

$$
\begin{aligned}
& a(x)=\left(x_{1}^{2}+x_{2}^{2}, 0\right)^{T}, b(x)=(0,1)^{T}, \\
& x_{1}(0)=-1, x_{2}(0)=0 .
\end{aligned}
$$

The equivalent control can be calculated here directly

$$
u_{e q}(t)=-(S b(x(t)))^{-1}[S a(x(t))]=0 .
$$

The corresponding sliding trajectory is contained into $\mathcal{S}$ that is characterized by the simple condition $x_{2}=0$. The solutions of the one dimensional differential equation $\dot{x}_{1}=x_{1}^{2}$ with $x_{1}(0)=-1$ is given by $x_{1}(t)=-1 /(t+1)$. Using the system Hamiltonian

$$
H(u, x, p)=p_{1}\left(x_{1}^{2}+x_{2}^{2}\right)+p_{2} u,
$$

where $p=\left(p_{1}, p_{2}\right)$, we can apply the formalism of Theorem 4. From (12) it follows:

$$
\dot{p}_{1}=-2 p_{1} x_{1}, \quad \dot{p}_{2}=-2 p_{1} x_{2}, \quad p_{2}=0
$$

and $-2 p_{1} x_{2}=0$. Under assumption $p \neq 0$ we obtain the sliding surface determined by the condition $x_{2}=0$. The sliding control that guarantee $x_{2}=0$ is the same $u_{e q}(t)=0$. The corresponding trajectory also coincides with the dynamic behavior obtained above $x_{1}(t)=-1 /(t+1), x_{2}(t)=0$.

\subsection{On the Degenerate and Non-degenerate Cases}

As mentioned above the system (12) from Theorem 4 is similar to the generic conditions of a weak Pontryagin Maximum Principle in the classic optimal control. From the proof of Theorem 4 it follows that the pair $\left(u_{e q}(\cdot), x(\cdot)\right)$ on $\left[T, t_{f}\right]$ can be formally considered as an optimal solution to 
the auxiliary optimal control problem on $\left[T, t_{f}\right]$ involved $(1)$ with the terminal costs functional $\phi\left(x\left(t_{f}\right)\right)$ such that

$$
\frac{\partial \phi}{\partial x}\left(x\left(t_{f}\right)\right)=p\left(t_{f}\right)=\rho^{T}, \quad \rho^{T} \Phi\left(t_{f}\right) \Phi^{-1}(t) b(x(t))=0,
$$

where $\rho \in \mathbb{R}^{n}, \rho \neq 0$.

From the point of view of the Hamiltonian system (12) the information about a sliding manifold $\mathcal{S}$ is now presented by the vector function $p(\cdot)$. Our main variational result, namely, Theorem 4 does not exclude a possible zerosolution for the second equation in (12). In that case we have a degenerate Hamiltonian system (12) characterized by $p(t) \equiv 0$. Let us illustrate this situation by the next simple example.

Example 2. Consider the following controllable linear system

$$
\dot{x}_{1}=x_{2}, \dot{x}_{2}=u
$$

with $x_{1}, x_{2}, u \in \mathbb{R}, t \in\left[T, t_{f}\right]$. The sliding manifold $\mathcal{S}$ associated with the system is assumed to be given by the condition $x_{1}+x_{2}=0$. The differential equation for the adjoint variable from (12) with $H(x, p)=p_{1} x_{2}+p_{2} u$ implies

$$
\dot{p}_{1}=0, \dot{p}_{2}=-p_{1} \text {. }
$$

From the Hamiltonian stationarity condition $(\partial H / \partial u=0)$ in $(12)$ we deduce that $p_{2}=0$. Therefore, we also have $p_{1}=0$. Otherwise, the equivalent control computed by the explicit formula (3) implies $w_{\text {eq }}(x)=-x_{2}$. Summarizing, we can note that in this case of the degenerate Hamiltonian system the conditions (12) are non-constructive and the equivalent control can not be found from the system (12).

Recall that the case of a degenerate Hamiltonian system (12) is similar to the analogous situation in the classic optimal control. When solving conventional optimal control problems based on some necessary conditions for optimality one is often faced with two technical difficulties: the irregularity of the Lagrange multiplier associated with the state constraint [20,41] and the degeneracy phenomenon (see e.g., [3]). Various supplementary conditions (so called constraint qualifications) have been proposed under which it is possible to assert that the Lagrange multiplier rule holds in a "usual" 
constructive form (see [20]). Examples are the well known Slater regularity condition for classic convex programming and the Mangasarian-Fromovitz regularity conditions for general nonlinear optimization problems. We refer to $[5,3,18,23,33,41]$ for details. Note that some regularity conditions for constrained optimal control problems can be formulated as controllability conditions for the linearized system [33]. Our aim now is to formulate some conditions that guarantee the existence of a non-degenerate $(p(t) \neq 0)$ solution of the main Hamiltonian system in (12). We firstly recall the following general concept of a end-point mapping (see e.g., [19]).

Definition 1. Consider system (1) and assume that all the conditions from Section 2 are satisfied. The mapping $E\left(x_{0}, t_{f}\right): \mathcal{U} \rightarrow x\left(t_{f}\right)$ is called an end-point mapping associated with this system.

Note that $E\left(x_{0}, t_{f}\right)$ is defined here for fixed $x_{0}, t_{f}$. The differentiability properties of this mapping in the context of some applications are studied in [19]. We only mention here the following useful fact.

Proposition 2. Under the basic conditions of Section 2 the end-point mapping $E\left(x_{0}, t_{f}\right)$ is Frechet differentiable and the corresponding derivative $E_{u}\left(x_{0}, t_{f}\right)$ at $u(\cdot) \in \mathcal{U}$ can be computed as follows: $E_{u}\left(x_{0}, t_{f}\right)=\delta x\left(t_{f}\right)$, where $\delta x(\cdot)$ is a solution to the linearized system (10). If the Frechet derivative $E_{u}\left(x_{0}, t_{f}\right)$ of the end-point mapping $E\left(x_{0}, t_{f}\right)$ is a non-surjective, then the Hamiltonian system in (12) is non-degenerate and there exists an absolute continuous solution $p(\cdot)$ to the adjoint equation from (12) with $p(t) \in \mathbb{R}^{n} \backslash\{0\}$.

As we can see, Proposition 2 express a sufficient condition for a nondegeneracy of the Hamiltonian system under consideration. It is easy to see, that for the linear version of the basic systems (1) with

$$
a(x)=A \in \mathbb{R}^{n \times n}, \quad b(x)=B \in \mathbb{R}^{n \times m}
$$

the standard controllability condition guarantees the surjectivity of the Frechet derivative $E_{u}\left(x_{0}, t_{f}\right)$ of the associated end-point mapping. Therefore, these systems are in the bad set and indeed they correspond to the degenerate 
Hamiltonian systems (12) (as shown in Example 2). However, one can explicitly use the given information on the sliding surface (the algebraic invariant) $\mathcal{S}$ and introduce the "regularized" pseudo-Hamiltonian

$$
\mathcal{H}(u, x, p):=\langle p, a(x)+b(x) u\rangle-\frac{1}{2}\|S x+c\|^{2}
$$

that corresponds to the original system (1) augmented by the following artificial differential equation for the new coordinate $x_{+1}: \dot{x}_{n+1}=\frac{1}{2}\|S x+c\|^{2}$. It is common knowledge that $\mathcal{H}(u, x, p)$ can also be interpreted as a Hamiltonian in the following Bolza type optimal control problems with terminal and integral terms

$$
\begin{aligned}
& \operatorname{minimize} \phi\left(t_{f}\right)+\frac{1}{2} \int_{0}^{t_{f}}\|S x(t)+c\|^{2} d t \\
& \text { subject to }(1), t \in\left[T, t_{f}\right], u(\cdot) \in \mathcal{U} .
\end{aligned}
$$

Evidently, the above-mentioned augmentation of the initial system (1) or the equivalent consideration of the optimal control problem (16) with the Bolza costs functional involves a necessary "information" regarding the existent algebraic invariant in an explicit form. In that case the proof of our main Theorem 4 can also be realized for the extended Hamiltonian $\mathcal{H}(u, x, p)$ without any conceptual changes. Using the same orthogonality argument and the existence of the algebraic invariant for the system (1) one can claim the existence of a vector $\rho \in \mathbb{R}^{n}$ such that

$$
\rho^{T}\left[\Phi\left(t_{f}\right) \Phi^{-1}(t) b(x(t))-(S x(t)+c)^{2}\right]=0
$$

for almost all $t \in\left[T, t_{f}\right]$. Recall that $x(t) \in \mathcal{S}$ for $t \in\left[T, t_{f}\right]$ and the formal augmentation above is in fact an augmentation by an effective zero. The last condition implies the modified adjoint equation similar to the second equation in (12) with respect to the augmented Hamiltonian $\mathcal{H}(u, x, p)$. Let us apply the proposed technique to the system from Example 2 with the initially degenerate Hamiltonian system.

Example 3. Consider the dynamic model from Example 2 and extend this original system by the auxiliary equation

$$
\dot{x}_{3}=\frac{1}{2}\left(x_{1}+x_{2}\right)^{2} .
$$


The augmented Hamiltonian has the following form:

$$
\mathcal{H}=p_{1} x_{2}+p_{2} u+p_{3} \frac{1}{2}\left(x_{1}+x_{2}\right)^{2} .
$$

From (12) we deduce

$$
\dot{p}_{1}=-p_{3}\left(x_{1}+x_{2}\right), \dot{p}_{2}=-p_{1}\left(x_{1}+x_{2}\right), \dot{p}_{3}=0
$$

and $p_{2}=0$. The obtained adjoint system has a non-zero solution $\left(p_{1}, 0, p_{3}\right)$ with some constant $p_{1}$ and $p_{3}$ if $x_{1}+x_{2}=0$. That leads to the correct characterization of the equivalent control $u_{e q}(t)=x_{1}(t)=-x_{2}(t), t \in\left[T, t_{f}\right]$.

Note that the linearized system (10) for the given augmented system in Example 3 is a controllable system. On the other hand, the surjectivity condition in Proposition 2 is only a sufficient condition for the non-degeneracy of the Hamiltonian system in (12).

\section{The Numerical Aspects}

Let us now discuss a computational scheme for a concrete evaluating the extremal controls determined by (12). For a nonlinearly affine system of the type (1) the extremal condition for the Hamiltonian in (12) implies the following

$$
\left\langle p(t), F_{j}(x(t))\right\rangle=0 \quad \forall j=1, \ldots, m \text { a.e. on }\left[T, t_{f}\right] .
$$

Differentiating (17) with respect to $t$, we get the equation

$$
q(x(t), p(t))+Q(x(t), p(t)) u_{e q}(t)=0,
$$

where $q(x, p)$ is the $m$-dimensional vector with components

$$
q_{j}(x, p):=\left\langle p,\left[F_{j}, a\right](x)\right\rangle
$$

and $Q(x, p)$ is the $m \times m$ skew-symmetric matrix with

$$
O_{i, j}:=\left\langle p,\left[F_{i}, F_{j}\right](x)\right\rangle .
$$

By $[\cdot, \cdot]$ we denote here the Lie brackets defined by the usual convention:

$$
\left[Z_{1}, Z_{2}\right](\xi):=\frac{\partial Z_{1}}{\partial \xi}(\xi) Z_{2}(\xi)-\frac{\partial Z_{2}}{\partial \xi}(\xi) Z_{1}(\xi)
$$


Clearly, (18) has a constructive character only in the case $\left[F_{j}, a\right](x) \neq 0$ and $\left[F_{i}, F_{j}\right](x) \neq 0$ for all $x \in \mathbb{R}^{n}$ and all $i, j=1, \ldots, m$. This relation represents a constructive basis for the constructive numerical treatment of our main Theorem 4.

Consider system (1) and suppose that $m \in \mathbb{N}$ is even. Moreover, assume that $\max _{(x, p)} \operatorname{rank} Q(x, p)=m$ and $\operatorname{det}\{Q(x, p)\} \neq 0$ for the all pairs $(x, p)$ that satisfy (18). Under these additional assumptions relation (18) implies

$$
u_{e q}(t)=-Q^{-1}(x(t), p(t)) q(x(t), p(t))
$$

Here $x(\cdot)$ and $p(\cdot)$ are calculated by (12). For an odd number $m \in \mathbb{N}$ one can obtain a similar formula, the only complication being the existence of a kernel for $Q(x, p)$. In that case one can extend the original system (1) by an additional differential equation of the type $\dot{x}_{n+1}=u_{m+1}$ such that the rank conditions (from Section 2) for the newly defined $\operatorname{span}\left\{F_{1}(x), \ldots, F_{m}(x)\right\}$ are also satisfied. In such a way we evidently come back to the case of an even control dimension.

Let us now apply the numerical idea discussed above to an illustrative computational example.

Example 4. Consider the nonlinear system

$$
\left\{\begin{array}{c}
\dot{x}_{1}=x_{1}^{2}+x_{2}^{2}+x_{1} u_{1} \\
\dot{x}_{2}=x_{1}^{2}+x_{2} u_{2}
\end{array}\right.
$$

with some suitable initial conditions $x_{1}(0)$ and $x_{2}(0)$. Assume that the sliding manifolds $\mathcal{S}_{1}$ and $\mathcal{S}_{2}$ are expressed by $x_{1}+x_{2}=0$ and $x_{1}-x_{2}$, respectively. The corresponding initial conditions for these two cases are assumed to be nontrivial and moreover, $x_{1}(0)+x_{2}(0)=0$ or $x_{1}(0)-x_{2}(0)=0$. It is easy to see that the given control strategy

$$
\left(\hat{u}_{1}(\cdot), \hat{u}_{2}(\cdot)\right), \hat{u}_{1}(t):=-3 x_{1}(t), \hat{u}_{2}(t) \equiv 0,
$$

implies the sliding condition $x(t) \in \mathcal{S}_{1}$ for all $t \geq 0$. For $x(t) \in \mathcal{S}_{2}$ we get the next control strategy

$$
\left(\tilde{u}_{1}(\cdot), \tilde{u}_{2}(\cdot)\right), \quad \tilde{u}_{1}(t):=-x_{1}(t), \hat{u}_{2}(t) \equiv 0 .
$$

Note that control pairs $\left(u_{1}(\cdot), u_{2}(\cdot)\right)$ that guarantees the above two sliding behaviors are non uniquely defined. For example, the condition $x(t) \in \mathcal{S}_{2}$ 
can also be guaranteed for the given control system closed by $u_{1}(t)=0$ and $u_{2}(t)=x_{2}(t)$. Here we use the natural notation $x(t):=\left(x_{1}(t), x_{2}(t)\right)^{T}$. The first components of the obtained control pairs are calculated using the explicit equivalent control formalism (3).

We now characterize the same sliding dynamic behavior by the Hamiltonian system (12). Evidently,

$$
H(u, x, p)=p_{1}\left(x_{1}^{2}+x_{2}^{2}+x_{1} u_{1}\right)+p_{2} x_{1}^{2}+p_{2} x_{2} u_{2}, p:=\left(p_{1} p_{2}\right)^{T}
$$

and we easily obtain the corresponding adjoint system

$$
\left\{\begin{array}{c}
\dot{p}_{1}=-\left(2 p_{1} x_{1}+p_{1} u_{1}+2 p_{2} x_{1}\right) \\
\dot{p}_{2}=-\left(2 p_{1} x_{2}+p_{2} u_{2}\right)
\end{array}\right.
$$

The last condition in (12) implies the algebraic condition $\left(p_{1} x_{1}, p_{2} x_{2}\right)^{T}=0$. Since the assumption $x_{2}=0$ implies $x_{1}=0$, we get the contradiction with the non-triviality of the initial conditions. Therefore, $p_{2}=0$. Differentiating the expression $p_{1} x_{1}=0$ with respect to the time and replace the derivatives of the adjoint variable using (20), we get the simple orthogonality condition.

$$
p_{1}\left(x_{1}-x_{2}\right)\left(x_{1}+x_{2}\right)=0 .
$$

From the assumption $P \neq 0$ it follows the characterization of the possible sliding manifolds $\mathcal{S}_{1}$ and $\mathcal{S}_{2}$. Note that the formalism (19) can be obtained applying the initially given system. For example, in the case $x_{1}=x_{2}$ we use the given information about the derivatives (from the dynamic system) and derive the expression $u_{1}-u_{2}=-x_{1}$. Clearly, the specific control pair $\left(\tilde{u}_{1}(\cdot), \tilde{u}_{2}(\cdot)\right)$, where $\tilde{u}_{1}(\cdot)$ is an equivalent control, satisfies the obtained general relation between the control components for the case $x(t) \in \mathcal{S}_{2}$.

Let us finally note that our main theoretic result, namely, Theorem 4 as well as the corresponding numerical tool (formula (18)) does not use the dimensionality restriction $\operatorname{dim} \mathcal{S}=m<n$ that is typical for the sliding mode control design. Therefore, Theorem 4 can be considered as a generalization of the equivalent control concept for the general case $\operatorname{dim} \mathcal{S}<n$. 


\section{Concluding Remarks}

In this paper, we have initiate the theoretic investigations devoted to a Hamiltonian based variational approach to some classes of the conventional sliding mode control processes. The main idea of the proposed approach constitutes an extension of the specific approximation techniques and an application of the Hamiltonian formalism. The proposed constructive approximations and the resulting variational description have a general nature and can be also applied to wide classes of dynamic models with discontinuous right hand sides. For control systems with affine structure closed by a typical sliding mode feedback synthesis we also propose a specific approximating differential inclusion. This new mathematical object can be characterized as an theoretic extension of the classic Filippov approach. The considered variational representation of the class of variable structure systems incorporates the standard Hamiltonian system and some associated extremal conditions. We have obtained a new specification of the sliding trajectory in the context of a solution to an auxiliary optimal control problem. The last one has a potential to be constructively implemented and applied to a concrete sliding mode type control design procedure.

The methodology discussed in this paper can be developed not only to the classical sliding mode dynamics but also to hight-order realizations. In that case the generic variational Hamiltonian-based description needs to be extended by additional state/derivatives constraints related to the admissible hight-order sliding mode feedback control law. These restrictions are usually given in the following form

$$
\omega(x):=\tilde{\omega}\left(\sigma(x), \dot{\sigma}(x), \ldots, \sigma^{(l-1)}(x)\right) .
$$

Here $l \in N$ is a relative degree of the system under consideration. The resulted high order design procedure constitutes a sophisticated problem that incorporates not only the usual state constraint $\sigma(x(t))=0$ but is also a set of additional algebraical constrains

$$
\dot{\sigma}(x(t))=0, \ldots \sigma^{(l-1)}(x(t))=0 .
$$

Under some concrete restrictions the specific structure of the above-mentioned feedback control law can guarantee so called "finite-time" stability of the corresponding closed-loop system. A formal consequence of this dynamic property is a singularity of the end point mapping. This fact implies a possibility to rewrite the closed-loop model in an equivalent Hamiltonian form. 
The initial analytic results discussed in our contribution need to be extended by some concrete control applications. Moreover, we also expect to obtain some numerically tractable approximation schemes and solution procedures for the sliding mode design. Finally note that the mathematical tools and analytic techniques developed in our paper can provide a new methodology for some control problems associated with the general classes of switched and hybrid control systems (see [6, 7, 9, 42]). It seems to be possible apply the elaborated theoretic approach to the description of the Zeno-type dynamic processes [2].

\section{References}

[1] C.D. Aliprantis and K.C. Border, Infinite Dimensional Analysis, Springer, Berlin, 1999.

[2] A. Ames and S. Sastry, Blowing up hybrid affine systems, in: Proceedings of the 2004 Conferenc on Decision and Control, Atlantis, Bahamas, 2004, pp. $473-478$.

[3] A.V. Arutyunov and S.M. Aseev, Investigation of the degeneracy phenomenon in the maximum principle for optimal control with state constraints, SIAM Journal on Control and Optimization, vol. 35, 1997, pp. $930-952$.

[4] U.M. Ascher, H. Chin and S. Reich, Stabilization of DAEs and invariant manifolds, Numerische Mathematik, vol. 67, 1994, pp. 131 - 149.

[5] V. Azhmyakov and J. Raisch, Convex control systems and convex optimal control problems with constraints, IEEE Transactions on Automatic Control, vol. 53, 2008, pp. $993-998$.

[6] V. Azhmyakov, V.G. Boltyanski and A. Poznyak, Optimal control of impulsive hybrid systems, Nonlinear Analysis: Hybrid Systems, vol. 2, 2008, pp. 1089 - 1097.

[7] V. Azhmyakov, R. Galvan-Guerra and M. Egerstedt, Hybrid LQoptimization using Dynamic Programming, in: Proceedings of the 2009 American Control Conference, St. Louis, USA, 2009, pp. 3617 - 3623. 
[8] V. Azhmyakov, M. Egerstedt, L. Fridman and A. Poznyak, Approximability of nonlinear affine control systems, Nonlinear Analysis: Hybrid Systems, vol. 5, 2011, pp. 275 - 288.

[9] V. Azhmyakov, M.V. Basin and J. Raisch, A proximal point approach to optimal control of affine switched systems, Discrete Event Dynamical Systems, vol. 22, 2012, pp. $61-81$.

[10] V. Azhmyakov, On the set-valued approach to optimal control of sliding mode processes, Journal of the Franklin Institute, vol. 349, 2012, pp $1323-1336$.

[11] V. Azhmyakov, On the geometric aspects of the invariant ellipsoid method: application to the robust control design, in: Proceedings of the 2011 Conference on Decision and Control, Orlando, USA, 2011, pp. $1353-1358$.

[12] G. Bartolini, L. Fridman, A. Pisano and E. Usai (Eds.), Modern Sliding Mode Control Theory, Lecture Notes in Control and Information Sciences, vol. 375, Springer, Berlin, 2008.

[13] M.V. Basin, A. Ferrara, and D. Calderon-Alvarez, Sliding mode regulator solution to optimal control problem, in Proceedings of the 4 th Conference on Decision and Control, Cancun, Mexico, 2008, pp. 2184 2189.

[14] L.D. Berkovitz, Optimal Control Theory, Springer, New York, 1974.

[15] S. P. Bhat and D. S. Bernstein, Finite time stability of continuous autonomous systems, SIAM Journal on Control and Optimization, vol. 38, 2000, pp. $751-766$.

[16] I. Boiko, Discontinuous Control Systems Frequency-Domain Analysis and Design, Birkhauser, New York, 2009.

[17] I. Boiko, L. Fridman, A. Pisano and E. Usai, On the transfer properties of the "generalized sub-optimal" second-order sliding mode control algorithm, IEEE Transactions on Automatic Control, vol. 54, 2009, pp. $399-403$. 
[18] V.G. Boltyanski and A. Poznyak, Robust Maximum Principle, Birkhaeuser, Boston, 2011.

[19] B. Bonnard and M. Chyba, Singular Trajectories and their Role in Control Theory, Springer, Berlin, 2003.

[20] F.H. Clarke, Yu.S. Ledyaev, R.J. Stern and P.R. Wolenski, Nonsmooth Analysis and Control Theory, Springer, New York, 1998.

[21] K. Deimling, Multivalued Differential equations, de Gruyter, Berlin, 1992.

[22] H.O. Fattorini, Infinite-Dimensional Optimization and Control Theory, Cambridge University Press, Cambridge, UK; 1999.

[23] M.M.A. Ferreira, F.A.C.C. Fontes and R.B. Vinter, Nondegenerate necessary conditions for nonconvex optimal control problems with state constraints, Journal of Mathematical Analysis and Applications, vol. 233, 1999, pp. $116-129$.

[24] A. Ferrara and M. Rubbagoti A sub-optimal second order sliding mode controller for systems with saturating actuators, IEEE Transactions on Automatic Control, vol. 54, 2009, pp. 1082 - 1087.

[25] A.F. Filippov, Differential Equations with Discontinuous Right-Hand Sides, Kluwer, Dordrecht, 1988.

[26] L. Fridman, J. Davila and A. Levant, High-order sliding-mode observation for linear systems with unknown inputs, Nonlinear Analysis: Hybrid Systems, vol. 5, 2011, pp. 189 - 205.

[27] A. G. Gallardo-Hernandez, L. Fridman, S. Islas-Andrade, Y. Shtessel, Quasi-continuous high order sliding modes controllers applied to glucoseinsulin regulatory system models, in: Proceedings of the 47th IEEE Conference on Decision and Control, Cancun, Mexico, 2008, pp. 2208 2213.

[28] R. Gamkrelidze, Principles of Optimal Control Theory, Plenum Press, London, 1978.

[29] J. Hale, Ordinary Differential Equations J. Wiley, New York, 1969. 
[30] C.J. Himmelberg, Measurable relations, Fundamenta Mathematicae, vol. 87,1975 , pp. $53-72$.

[31] Y. Hong, Finite-time stabilization and stabilizability of a class of controllable systems, Systems and Control Letters, vol. 46, 2002, pp. 231 236.

[32] A. Isidori, Nonlinear Control Systems, Springer, New York, 1989.

[33] J. Jahn Introduction to the Theory of Nonlinear Optimization. Springer, Berlin, 2007.

[34] H.K. Khalil, Nonlinear Systems, Prentice Hall, Upper Saddle River, 2002.

[35] A. Levant, Universal SISO sliding-mode controllers with finite-time convergence, IEEE Transactions on Automatic Control, vol. 46, 2001, pp. $1447-1451$.

[36] Z. Lin, Y. Xia, P. Shi and H. Wu, Robust sliding mode control for uncertain linear discrete systems independent of time-delay, International Journal of Innovative Computing Information and Control, vol. 7, 2011, pp. $869-881$.

[37] E. Moulay and W. Perruquetti, Lyapunov based approach to finite-time stability and stabilization, in: Proceedings of the 44 th Conference on Decision and Control, seville, Spain, 2005, pp. 4742 - 4747.

[38] Y. Orlov, Discontinuous Systems: Lyapunov Analysis and Robust Synthesis under Uncertainty Conditions, Springer, New York, 2008.

[39] B.E. Paden and S.S. Sastry, Calculus for computing Filippov's differential inclusion with application to the variable structure control of robot manipulator, IEEE Transactions on Circuits Systems, vol. 34, 73 - 82, 1987.

[40] A. Polyakov and A. Poznyak, Reaching time estimation for "supertwisting" second order sliding mode controller via Lyapunov function designing, IEEE Transactions on Automatic Control, vol. 54, 2009, pp. $1951-1955$. 
[41] A. Poznyak, Advanced Mathematical Tools for Automatic Control Engineers. Elsevier, Amsterdam, 2008.

[42] M.S. Shaikh and P. E. Caines, On the hybrid optimal control problem: theory and algorithms, IEEE Transactions on Automatic Control, vol. 52, 2007, pp. $1587-1603$.

[43] V. Utkin, Sliding Modes in Control and Optimization. Springer, Berlin, 1992.

[44] V. Utkin, A. Poznyak and P. Ordaz, Adaptive super-tvist control with minimal chattering effect, in: Proceedings of the 2011 Conference on Decision and Control, Orlando, USA, 2011, to appear.

[45] L. Wu, P. Shi and H. Gao, State estimation and sliding mode control of Markovian jump singular systems, IEEE Transactions on Automatic Control, vol. 55, 2010, pp. $1213-1219$.

[46] H. Wu and P. Shi, Adaptive variable structure state estimation for uncertain systems with persistently bounded disturbances, International Journal of Robust and Nonlinear Control, vol. 20, 2010, pp. 2003 - 2015.

[47] Y. Xia, H. Yang, M. Fu and P. Shi, Sliding mode control for linear systems with time-varying input and state delays, Circuits Systems and Signal Processing, vol. 30, 2011, pp. 629 - 641.

[48] W. Xiang and F. Chen, Sliding mode control strategies for the hyperchaotic MCK system, ICIC Express Letters, vol. 3, 2009, pp. 283 288.

[49] L. Yu, S. Fei, H. Zu and X. Li, Direct adaptive neural control with sliding mode method for a class of uncertain switched nonlinear systems, International Journal of Innovative Computing, Information and Control, vol. 6, 2010, pp. $5609-5618$.

[50] M. Zhang, Z. Yu, H. Huan and Y. Zhou, The sliding mode variable structure control based on composite reaching law of active magnetic bearing, ICIC Express Letters, vol. 2, 2008, pp.59 - 63. 